\title{
Deformation profile of a piece of japonica cooked rice with squeezing test
}

\author{
Efrina Efrina ${ }^{1,2, *}$, Yoshiyuki Komoda ${ }^{1}$,Hiroshi Suzuki ${ }^{1}$, and Ruri Hidema ${ }^{3}$ \\ ${ }^{1}$ Kobe University, Department of Chemical Science and Engineering, Kobe, Japan \\ ${ }^{2}$ State University of Jakarta, Department of Home Economics, Faculty of Engineering, Jakarta, Indonesia \\ ${ }^{3}$ Kobe University, Organization of Advanced Science and Technology, Kobe, Japan
}

\begin{abstract}
The texture of cooked rice plays an important role in the characteristics of cooked rice. It can be firmness or stickiness depends on the amount of amylopectin inside the cooked rice, the higher the content will be more stickiness. Japonica rice is the type of rice with highest amylopectin content. Therefore the texture is the most stickiness. This research is to define the stickiness of japonica cooked rice using stressstrain behavior through uniaxial compression test. Stress defined as a normal force divided by a crosssectional area in the horizontal plane while strain as a shrinking ratio of cooked rice. A single Japonica cooked rice squeezed using parallel plate type rheometer with constant velocity $(0,2 ; 0,5 ; 1 ; 2 ; 4 \mathrm{~mm} / \mathrm{s})$. The deformation process of cooked rice was recorded from both side and bottom views to measure area transformation. The result showed that stress-strain of the deformation of cooked japonica rice was strongly depended on velocity. The low velocity $(0,2 ; 0,5 ; 1$ and $1 \mathrm{~mm} / \mathrm{s})$ are same with high velocity $(2$ and $4 \mathrm{~mm} / \mathrm{s})$ the during first deformation but significantly different statistically in the later deformation.
\end{abstract}

\section{Introduction}

Rice is divided into two major subspecies based on their appearance, aroma, taste, hardness, stickiness and chemical ingredients which are Indica Rice (long grain) and Japonica Rice (short grain) [1]. These grains showed different chemical, especially on amylose and amylopectin content. Amylose and amylopectin detected the most important content that affected the texture of cooked rice. The higher the amylopectin content, it is more stickiness [2-4]. Japonica cooked rice is the highest amylopectin content so that its texture is stick and glossy.

The texture of food product can be different for each person [5]. Definition of the firm or soft can be different as everyone has a different feeling [6].There is some factors, such as the presence of pain and temporomandibular disorders, gender, age, cranio-facial morphology, and occlusal that affected to bite force during eating [7]. The exact processes during food intake are not fully understood because it included several process and action that belong to several fields of study such as food, psychology, physiology, dental and clinical studies. Quantitative expression of material deformation helps scientists to understand more clearly about the relation between texture and oral processing. It is reported that food stuff is sensitive to the stressapplication velocity and that its texture can be described by mechanical properties during deformation process [8]. There are some evidences for measuring mechanical properties especially texture with some instrument

\footnotetext{
*Corresponding author: efrina@unj.ac.id
}

according to the motion they used to deform the food, which are linear, rotary and combined linear and rotary [9]. More over some evidence showed that velocity is played an important role in defining texture during first biting. Velocity $0.2-12 \mathrm{~mm} / \mathrm{s}$ are the velocity which showed the same velocity during first biting and mastication [10].

In this study, we examined the deformation process of cooked rice within squeezing plates at several velocities and measured the changed of the cross-sectional area during deformation. The squeezing flows are flows which a material deformed between two parallel plates [11]. The squeezing flow can be model at some phenomena during food intake. Two parallel and material can approximate the contact between teeth-food-teethand deformation process can be calculated. Large deformation is analysed to show strain hardening in intermediate strain and strain softening near yield strain which is connected to stressstrain curve [12]. In this research, the upper plate will be moving down where the bottom plate will be fixed. The velocity of moving down upper plate is constant.

\section{Materials and Methods}

Japonica rice cooked by using a commercial rice cooker incorporated with 1.4 times amount of water in volume. Food coloring was added during cooking process to facilitate the calculation of the dimensions of cooked rice. A piece of cooked rice was then squeezed using a parallel plate type rheometer with a constant velocity $0.2 ; 0.5 ; 1$; 
$2 ; 4 \mathrm{~mm} / \mathrm{s}$. Deformation of cooked rice was recorded from both side and bottom views as illustrated in Fig.1. The sequence of the images during deformation was analyzed using ImageJ software to define area transformation. A cooked rice was illustrated as an ellipsoid having the dimensions of ellipsoid ( $x$ and $y$ ) and height $z$. The normal force is being recorded as a function of gap ( $\mathrm{z}$ axis), the distance between the plates. A logarithmic shrinking ratio on gap during deformation are stated as hencky strain [13]. While area defined as a cross-sectional area in the horizontal plane ( $\mathrm{x}$ and $\mathrm{y}$ axis).
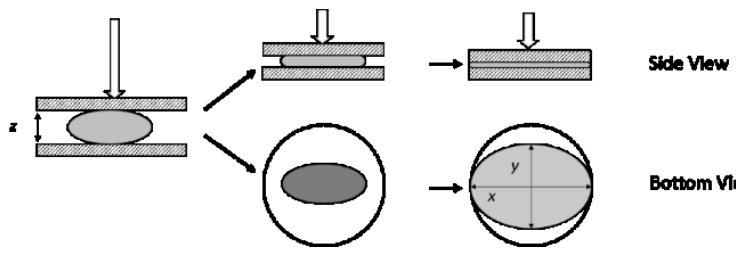

Bottom Vlew

Fig. 1 Schematic view of measurement Area.

\section{Results and Discussion}

During deformation of Japonica cooked rice, force increase slowly and rapidly on the end of deformation. By increasing trend of the cross-sectional area during deformation process as a function of strain (Fig 2), the area was increased slightly in the early stage of deformation or a small strain region $(\mathrm{eH})$, while it showed a drastic increase in the latter stage with larger strain.

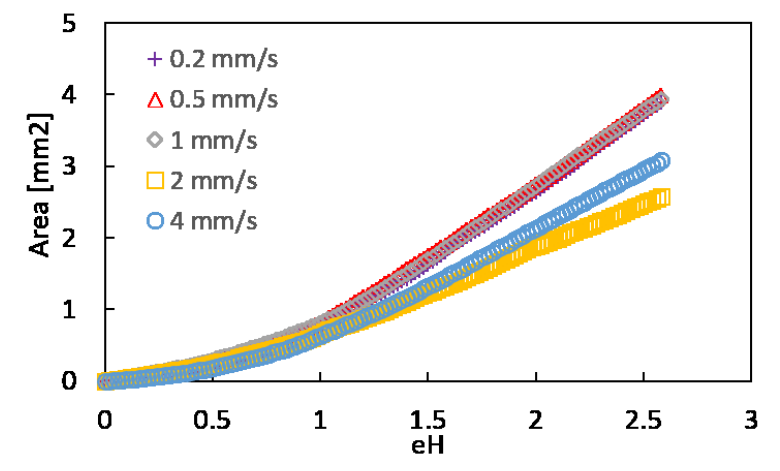

Fig 2. Cross-sectional area change as a function of henckystrain.

Fig 2 showed that all velocity have the same slope on early deformation, but significantly on the larger strain. By Man-Whitnney test, all populations on velocities are not significantly different on strain between $0-1$. On higher strain, the slopes are slightly changed between 0,2 ; 0,5 ; and $1 \mathrm{~mm} / \mathrm{s}$ while showed significantly different with higher velocity ( 2 and $4 \mathrm{~mm} / \mathrm{s}$ ). Therefore we decided to divide into two groups by its area transformation, low velocity group, and high velocity groups.

Same behavior showed on the relation between hencky strain and force (Fig 3). Man-Whitnney test showed that there are no significantly different between velocities on low velocity groups but have significantly different with high groups. Increasing cross-sectional area on food stuff affected by the force even though the stress produced did not change [14]. Increasing of the area on high velocity have a smaller slope than low velocity therefore on high velocity, force increased more rapidly while

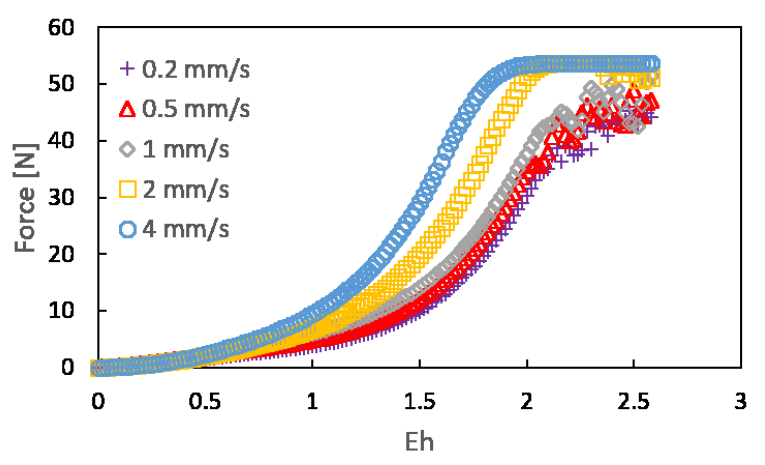

Fig 3.Increasing force curve to strain.

The small slope of the first stage indicates that a cooked rice tried to resist its structure from the stress applied by the moving-down upper plate. It testified that a cooked rice behaves as an elastic solid body. On low velocity the changing area of deformation are stages by the value of its slope. The first stage indicated no difference in slope at all velocities. In the last stage, slopes monotonously change at the velocity $2 \mathrm{~mm} / \mathrm{s}$ and more. The second stage started when a cooked rice could not maintain its shape anymore so that deformation happened. At this stage, cooked rice behaves as a plastic solid body. The last stage happened when a surface of a cooked rice started to break. At this stage, cooked rice has reached the end of elastic and plastic deformation ranges that lead to structural fracture. The transition of between stages was changed with velocities; the strain at the onset of stage 2 or 3 becomes smaller at a higher velocity.

In order to elucidate the effect of velocity on the change of bottom view of a cooked rice, we depicted the increasing curves of long and short axes of ellipse of the cross-sectional area as shown in Fig.4. The graphs indicated that the increasing curve of short axis (width) changed its slope at the transition strain for the area between stage 1 and 2, implying that the area change in the former stages was mainly affected by the deformation of short axis. In contrast, the cooked rice was deformed in both directions, resulting in a drastic expansion of the area.

Addition of the food coloring made the surface of a cooked rice colored red. Therefore, if the internal part of cooked rice came out at the surface in the deformation process, the cross-sectional surface consisted of red and white parts. Based on the behavior of red parts being colored black in Fig.3, we could see that white part appeared at the onset of stage 3 . It means that the surface structure of a cooked rice was started to break down at the last stage. The degree of compression during squeezing test indicates that the voids and cell walls on cooked rice have some effect on texture [15]. 




a) Velocity $0.2 \mathrm{~mm} / \mathrm{s}$

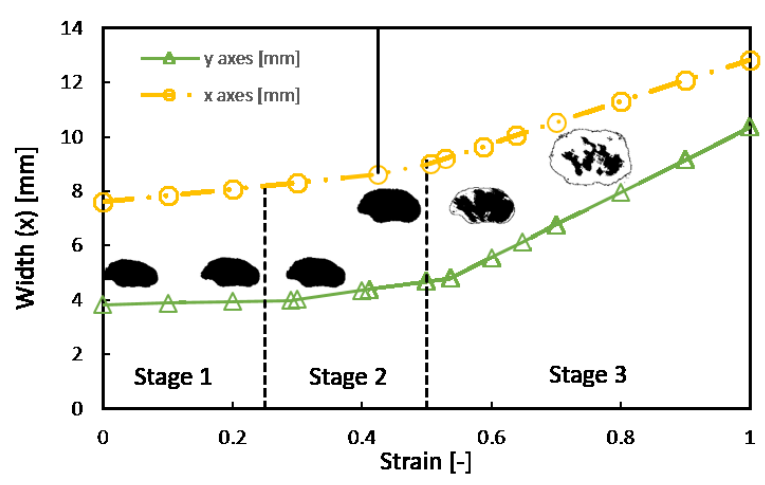

b) Velocity $4 \mathrm{~mm} / \mathrm{s}$

Fig. 3 Effect of squeezing velocity on the dimensions and surface structure of deformed cooked rice.

\section{Conclusions}

From this research, we concluded that the moving down the velocity of squeezing plate affect to strain-stress curve of japonica cooked rice. On the First stage, a cooked rice tried to resist its structure from the stress applied by the moving-down upper plate. A cooked rice behaves as elastic body. On Second stage, a cooked rice could not maintain its shape anymore so that deformation happened. A cooked rice behave as plastic body. Moreover, during the last stage, a cooked rice deformed exponentially. It assumed that the stickiness of cooked rice plays an important role in this stage

\section{Reference}

1. FAO. Rice International Commodity Profile. Mark Trade Div (2006)

2. D.K Cameron, Y.J Wang. Cereal Chem. 82(2): 1139 (2005)

3. L.Yang, Y.H. Sun, Y. Liu, Q.Mao, L.X.You, J.M Hou, M.A.Ashraf. Brazilian Arch Biol Technol. 59 (2016)

4. L.B. Iturriaga, B.L de Mishima, M.C. Añon. Food Res Int. 39 (2006)

5. M. Bourne. Food texture and Viscosity 2nd ed. (2002)
6. M.C. Bourne. J Food Sci 32 (1967)

7. D. Koc, A. Dogan, B.Bek. Eur J Dent 4 (2010)

8. C. Wilkinson, G.B. Dijksterhui, Minekus M. Trends Food Sci. Technol. 11 (2000)

9. P.W. Voisey PW. Rheology and Texture in Food Quality (1976)

10. K. Kohyama, A. Nagata, Y. Tamaki, N. Sakurai . Postharvest Biol Technol. 52 (2009)

11. J. Engmann, C. Servais, A.S. Burbidge. J Nonnewton Fluid Mech 132 (2005)

12. S. Kawai, Y. Nitta, K. Nishinari . J Chem Phys 128 (2008)

13. A.H. Chen, J.W. Larkin, C.J. Clark, W.E. Irwin. J Dairy Sci 62 (1979)

14. K. Kohyama, T. Sasaki, F. Hayakawa, E. Hatakeyama. Biosci Biotechnol Biochem 68 (2004)

15. Y. Ogawa, D.F. Wood, L.C. Whitehand, W.J. Orts, G.M. Glenn. Cereal Chem 83 (2006) 